\title{
The Gutenberg health study: associations between occupational and private stress factors and work-privacy conflict
}

Susan Garthus-Niegel ${ }^{1,2^{*}}$, Janice Hegewald ${ }^{1}$, Andreas Seidler ${ }^{1}$, Matthias Nübling ${ }^{3}$, Christine Espinola-Klein ${ }^{4}$, Falk Liebers ${ }^{5}$, Philipp S. Wild ${ }^{4,6,7}$, Ute Latza $^{5}$ and Stephan Letzel $^{8}$

\begin{abstract}
Background: Work-privacy conflict (WPC) is no longer a rarity but constitutes a societal problem. The objectives of the present study were (1) to investigate the distribution and prevalence of WPC among the employed participants in the Gutenberg Health Study at baseline and (2) to study the dependence of WPC on a broad range of private life and occupational characteristics as well as on psychosocial working conditions.

Methods: This analysis is based on a representative, population-based sample of 3,709 employees participating in the Gutenberg Health Study. Descriptive and bivariable analyses were carried out separately for women and men. Distribution and prevalence of WPC were examined according to socio-demographic and occupational characteristics as well as psychosocial working conditions. Further, stepwise selection of Poisson log-linear regression models were performed to determine which socio-demographic and occupational characteristics were most associated with the outcome variable WPC and to obtain adjusted prevalence ratios from the final model. The multivariable analyses were conducted both separately for women and men and with all subjects together in one analysis.

Results: There was a high prevalence of WPC in the present study $(27.4 \%$ of the men and $23.0 \%$ of the women reported a high or very high WPC). A variety of factors was associated with WPC, e.g. full-time employment, depression and many of the psychosocial risk factors at work. Also, the multivariable results showed that women were of higher risk for a WPC.

Conclusions: By affecting the individual work life, home life, and the general well-being and health, WPC may lead to detrimental effects in employees, their families, employers, and society as a whole. Therefore, the high prevalence of WPC in our sample should be of concern. Among women, the risk for suffering from WPC was even higher, most likely due to multiple burdens.
\end{abstract}

Keywords: Work-privacy conflict, Private life characteristics, Occupational characteristics, Psychosocial working conditions, Gender differences, Gutenberg Health Study

\footnotetext{
* Correspondence: susan.garthus-niegel@tu-dresden.de

${ }^{1}$ Institute and Policlinic of Occupational and Social Medicine, Faculty of

Medicine, TU Dresden, Fetscherstr. 74, D-01307 Dresden, Germany

${ }^{2}$ Department of Psychosomatics and Health Behaviour, Norwegian Institute

of Public Health, Oslo, Norway

Full list of author information is available at the end of the article
} 


\section{Background}

Numerous theories have been used to understand the intersection of work and family life [1]. The spillover theory $[2,3]$ suggests that experiences in the work domain spill over and affect experiences in the family domain (or vice versa) and has led to a large body of research examining the work-life interface. Generally speaking, work-life interface (or work-life interaction) refers to experiences in the work (family) domain that impact experiences in the family (work) domain [1]. These mutual influences may be both, positive or negative. However, the main research focus has been on the conflicts that may occur between work and family roles $[1,4]$. This negative focus may be understood against the background of the changing working conditions in the Western world over the last decades, such as the increase in service industry work $[5,6]$. Demands on the employees have also been changing. Flexibility regarding time and location, resiliency and social competency are increasingly becoming key qualifications. Further, the subjective indicator of work intensity, which describes workers' experience of high demands, reveals an overall increase in work intensity in most European countries over the past two decades [5]. Dual-career couples and single-parent households find themselves struggling to juggle the competing demands of work and their private lives, which may result in an inter-role conflict $[7,8]$.

Various concepts have been used to describe such inter-role conflict. One common concept is the socalled "work-family conflict" (WFC) or "family-work conflict" (FWC). Also, "work interference with family" (WIF) and "family interference with work" (FIW) have been used synonymously [7]. A valid critique to these terms is that they cover only a segment of the labour force, namely those working women and men who have children or minors living at home. Therefore, it has been suggested to rather use the concept "worklife conflict" in order to also include singles, single parents, dual-income couples without children living at home etc. [9]. "Work-life conflict" however implies that work is not part of the employee's life, which is naturally not reflecting reality. Therefore, in the current study we choose to apply the term "workprivacy conflict" as we use the COPSOQ scale with the identical name measuring inter-role conflict. This term provides a precise distinction between both life areas and at the same time pertains to the entire private life.

Regarding WPC, two causal directions are conceivable: On the one hand, work-related stress factors such as shift work or precarious working conditions may have a negative impact on family and private life ("work-privacy conflict"). On the other hand, nonoccupational factors such as the personal life situation or major life events may conflict with work demands ("privacy-work conflict"). However, the former path appears to be more dominant and here the research evidence is the strongest $[9,10]$, probably also because the individual employee's suffering appears to be larger if the private life is affected than vice versa. Consequently, also the present paper relates to that direction.

In a recent meta-analysis, Byron (2005) examined key determinants of a WPC. Particularly work and nonwork variables (opposed to demographic variables such as sex and income) proved to be of importance. Job stress and hours spent at work increased the risk of suffering from a WPC, whereas a flexible schedule had a protective influence. Regarding the nonwork variables, both family stress and family conflict were important antecedents of a WPC [7].

In addition, there are various studies examining health-related effects of WPC [9]. Mental and physical health related outcomes included increased substance abuse (especially problem drinking), greater psychological stress, more frequent depression and other mental disorders, burnout, and other physical symptoms or somatic complaints including lack of appetite, sleep disorders, headaches or fatigue [9, 11-21]. However, results regarding objective health indices such as blood pressure and cholesterol level remain inconclusive [10].

Even though there are already various studies regarding WPC and associated factors, certain research gaps remain to be filled and further primary research has been called for $[7,9,10]$. In general, more European studies are warranted as most studies have been conducted in North America where working conditions are considerably different $[7,22$, 23]. More specifically, important work related variables like job level and job type as well as skill level have often not been assessed [7, 10]. In addition, the investigation of private life variables has been limited [10]. For instance, few studies differentiated between caring for children versus adults [7]; and previous research has mostly focused on conflicts between work and family, resulting in selective samples, namely those with employees who have children or minors living at home [9]. Samples in earlier studies have also been selective, because they mainly included middle-to upper-class employees [10].

In the current paper, we therefore examined WPC and a wide-ranging number of variables in a representative population-based sample of employees from the prospective Gutenberg Health Study (GHS). The objectives of the present explorative study were (1) to investigate the distribution and prevalence of WPC among the employed participants in the GHS at baseline and (2) to study the dependence of WPC on a broad range of private life and occupational characteristics as well as on psychosocial working conditions. 


\section{Methods}

\section{Study population}

The GHS is designed as a population-based, prospective, single-centre cohort study in the Rhine-Main region in Western Germany [24-26]. The primary aim is to evaluate and improve cardiovascular risk stratification. The GHS sample was drawn randomly from the governmental local registry offices in the city of Mainz and the district of Mainz-Bingen. The sample was stratified 1:1 for sex and residence (urban and rural) and in equal strata for decades of age. Between 2007 and 2012, 15,010 individuals between 35 and 74 years of age were enrolled, and written informed consent was obtained from all participants. Exclusion criteria were insufficient knowledge of the German language and physical or psychological inability to participate in the examinations at the study centre. Ethical approval of the study protocol and sampling design (including the present investigation) was given by the Ethics Commission of the State Chamber of Medicine in Rhineland-Palatinate and by the data protection officer of the University Medical Center of the Johannes Gutenberg University Mainz as well as the Rhineland-Palatinate data protection officer.

For the present study, we excluded subjects older than 64 years of age $(N=3,753)$. In order to analyse an unencumbered sample and to circumvent a "Healthy Worker Effect" we also excluded those with prevalent vascular diseases (i.e. coronary artery disease, myocardial infarction, stroke, peripheral vascular disease; $N=346$ ). In addition, 2,911 GHS participants were not eligible because they did not work and 236 participants had to be excluded because of missing data. A randomly selected sample $(N=4,055)$ filled in an alternative questionnaire regarding psychosocial working conditions (which did not contain questions related to WPC). The final sample in this study therefore consisted of 3,709 subjects (with 1,653 women and 2,056 men). There were fewer females (44.6\%) in the sample, as fewer women were employed than men. The mean age of the participants was 48 years (standard deviation $(\mathrm{SD})=7.5$ ).

For the current study, a completed STROBE checklist is provided as a supplementary file (see Additional file 1).

\section{Measures}

\section{Private life characteristics}

Age was measured as decades of age (i.e. 35-44, 45-54 and 55-64 years of age). Education was distinguished between school education ("certificate of secondary education (9th Grade)", "general certificate of secondary education (10th Grade)", “international baccalaureate (12th/13th grade)", "other certification" and "none") and occupational education ("vocational school/ apprenticeship", "technical school/ master craftsman", "university of applied sciences", "other qualification" and "none"). Socioeconomic status (SES) was measured using a multi-dimensional aggregated index [27]. The dimensions were "school and professional education", "occupation", and "income" and the resulting socioeconomic status groups were classified as "low", "intermediate" and "high". Marital status was classified as follows in brackets ("married", "registered partners", "divorced", "separated", "widow(er)" and "single, never married"). Further, the number of biological children ("0", " $1-2$ " and " $\geq 3$ "), the number of children below 18 years of age living at home ("0", "1-2" and " $\geq 3$ ") as well as the number of people living in the household ("1-2", "3-4" and " $\geq 5$ ") were measured. In addition it was assessed how much time was spent caring for children, caring for adult relatives, time spent on household errands, time spent on hobbies and time spent on job development ("0 h/week", "1-3 h/week" and " $\geq 4$ h/week" respectively). Smoking was assessed via both smoking status ("never", "quit 0-2 years ago", "quit more than 2 years ago" and "current") and pack years ("never smoked", "<20 pack years", "20-39 pack years" and " $\geq 40$ pack years"). Regarding alcohol intake TOAM limits (tolerable upper alcohol intake levels) [28] were used ("no intake"; "intake beneath tolerable limit" i.e., women $<=10 \mathrm{~g} /$ day, men $<=20 \mathrm{~g} /$ day; "intake above tolerable limit" i.e., women $>10-40 \mathrm{~g} /$ day, men $>20-60 \mathrm{~g} /$ day; and "abuse of alcohol" i.e., women $>40 \mathrm{~g} /$ day, men $>60 \mathrm{~g} /$ day). Depression was assessed by the Patient Health Questionnaire (PHQ-9) [29] via self-report (PHQ <10 "no depression" vs. $\mathrm{PHQ}>=10$ "depression").

\section{Occupational characteristics}

Occupations were manually double-coded according to the classification of occupations of the Federal Statistical Office Germany. The following occupational characteristics were assessed with the respective categories listed in brackets: form of employment (part-time vs. full-time employment), time spent at work ( $<40 \mathrm{~h} /$ week vs. $\geq 40 \mathrm{~h} /$ week $)$, night shift (yes/no), amount of night work (0-6 days/ month vs. $\geq 7$ days/month), job complexity level ("low" (helpers), "medium" (skilled workers), "complex" (specialists) and "very complex" (experts)), management (yes/no) and position ("worker", "employee", "government officials, judges, military employees", "self-employed/cooperative agriculturalist", "self-employed in trade, commerce, craftwork, industry, service", "academic self-employed profession (e.g. physician, attorney, tax consultant)", "student/ trainee" and "caretaker for relatives").

\section{Psychosocial working conditions}

The German version of the Copenhagen Psychosocial Questionnaire (COPSOQ) [30] was used to assess psychosocial working conditions. The COPSOQ consists of 5 thematic domains measuring 26 constructs (including WPC). The first four thematic domains represent the psychosocial factors at work: "Demands" (4 scales), "Influence and development" (5 scales), "Interpersonal relations and leadership" (9 scales) and "Further parameters" (1 scale in the present 
study: "insecurity at work"). The 5th domain represents "Strain" (6 constructs), assessing the reactions of the employees to the workplace situation as internal outcome parameters. The 6 "Strain" scales are "Job satisfaction", "Intention to leave", "General health", "Burnout", "Cognitive stress" and "Satisfaction with life".

\section{Work-Privacy Conflict (WPC)}

Just as with the nature of the construct, there is a lack of consistency with which WPC has been operationalized. Specific problems include the use of single-item measures and adapted and study-developed measures of unknown validity [10]. Allen and colleagues (2000) suggest to either use the WFC measure by Stephens and Sommer (1996), the WFC/FWC measure by Netemeyer (1996), or the WFC measure developed by Carlson, Kacmar, and Williams (1998) [31-33]. However, they favour the last one, as it includes all three forms of inter-role conflict (time-based, strain-based and behaviour-based) and measures both directions of an inter-role conflict [10].

In the present study we decided to use the "work-privacy conflict scale" (WPC scale), which is an established, validated scale and part of the COPSOQ thematic domain "Demands". It is assessed with 5 items using a 5point Likert scale, which can be combined and expressed as a percentage. We defined an indication of a WPC as a WPC Score of or exceeding $60 \%$ (this corresponds with the Likert-scale categories indicating high and very high WPC). The 5-item WPC Scale originates from the workfamily conflict scale by Netemeyer (1996), but was developed further for the COPSOQ to not only include family but the entire private life (changes are indicated in italics) [32]: 1. The demands of my work interfere with my private and family life; 2 . The amount of time my job takes up makes it difficult to fulfil family or private responsibilities; 3 . Things I want to do at home do not get done because of the demands my job puts on me; 4 . My job produces stress that makes it difficult to fulfil family duties; 5 . Due to work-related duties, I have to make changes to my plans for private or family activities. In all five items the coding of the Likert scale was as follows, 5="strongly agree", 4="agree", 3="undecided", $2=$ "disagree", $1=$ "strongly disagree". The WPC score was obtained by summing all five items and dividing by 5 . Reliability was excellent with $\alpha=0.91$.

\section{Statistical analysis}

Descriptive analyses were carried out separately for women and men. WPC was categorized as "very low" (<20 \%), "low" (20-39\%), "moderate" (40-59 \%), "high" $(60-79 \%)$ and "very high" (> $=80 \%)$. Also the bivariable analyses were carried out separately for women and men; distribution and prevalence of WPC were examined according to socio-demographic and occupational characteristics as well as psychosocial working conditions. All statistical analyses were performed in R [34]. For the regression analyses, WPC was dichotomized and defined as having a score of $>=60 \%$. Further, stepwise selection of Poisson stepwise log-linear regression models were performed to determine which sociodemographic and occupational characteristics were most associated with the outcome variable WPC and to obtain adjusted prevalence ratios (PR) from the final model. In order to examine the importance of working hours in more detail, we performed an additional sensitivity analysis where we instead of overtime ("Time spent at work $(40+\mathrm{h})$ ") and part-time employment included working hours per week. The multivariable analyses were conducted both separately for women and men and with all subjects together in one analysis.

For the stepwise selection, the stepAIC function of the MASS Package was used to determine models based on the Akaike information criterion (AIC) starting with a model that included only age (and sex in the combined model) [35].

\section{Results \\ Bivariable results \\ Private life characteristics}

More than half of the employed men reported to have a very low WPC (25.0\%) or low WPC (27.0\%) (see Table 1). Especially older men between 55 and 64 years of age had a very low WPC (34.9\%). $7.9 \%$ of all employed men reported to have a very high WPC. Particularly men with a university degree had a high $(24.8 \%)$ or very high WPC (10.4\%). Also men with a high SES (9.6 $\%)$, those without a hobby (9.3\%) und particularly those who did not spend time on household errands (19.4\%), had a very high WPC. Further, almost a fourth $(24.5 \%)$ of all men with depression had a very high WPC. Men who were divorced $(10.0 \%)$ or separated $(10.5 \%)$ were also more likely to report a very high WPC (Table 1).

Compared to men, the proportion of employed women who reported a very low WPC (34.0\%) was higher; $23.4 \%$ had a low WPC. Apart from that, distributions were similar to those of the men. Women aged 55-65 years tended to have a very low WPC (38.8\%), whereas a larger proportion of women with a university degree reported high (21.2\%) or very high WPC (9.6\%). Moreover, women with a high SES (11.1\%) und those not spending time on hobbies $(11.0 \%)$ more often had a very high WPC. Nearly a fourth $(21.6 \%)$ of all women with depression reported to have a very high WPC (Table 2).

\section{Occupational characteristics}

Regarding occupational characteristics, men who had to work night shifts, had a very high WPC (13.2 \%) (Table 3$)$. Also, men with highly complex tasks (10.1\%) 
Table 1 Private life characteristics and WPC among men

\begin{tabular}{|c|c|c|c|c|c|c|c|}
\hline & & & WPC n (\%) & & & & \\
\hline & Mean (SD) of WPC & $n$ & $\begin{array}{l}\text { Very low } \\
(<20 \%)\end{array}$ & $\begin{array}{l}\text { Low } \\
(20-39 \%)\end{array}$ & $\begin{array}{l}\text { Moderate } \\
(40-59 \%)\end{array}$ & $\begin{array}{l}\text { High } \\
(60-79 \%)\end{array}$ & $\begin{array}{l}\text { Very high } \\
(>=80 \%)\end{array}$ \\
\hline & & 2,056 & $514(25.0)$ & $555(27.0)$ & $424(20.6)$ & $401(19.5)$ & $162(7.9)$ \\
\hline Age & & & & & & & \\
\hline $35-44$ & $42(27)$ & 730 & $146(20.0)$ & $199(27.3)$ & $157(21.5)$ & $160(21.9)$ & $68(9.3)$ \\
\hline $45-54$ & $39(26)$ & 847 & $201(23.7)$ & $236(27.9)$ & $183(21.6)$ & $162(19.1)$ & $65(7.7)$ \\
\hline $55-64$ & $34(27)$ & 479 & $167(34.9)$ & $120(25.1)$ & $84(17.5)$ & 79 (16.5) & $29(6.1)$ \\
\hline Education (school) & & & & & & & \\
\hline $\begin{array}{l}\text { Certificate of Secondary } \\
\text { Education ( } 9^{\text {th }} \text { Grade) }\end{array}$ & $32(26)$ & 593 & $211(35.6)$ & $159(26.8)$ & $112(18.9)$ & $80(13.5)$ & $31(5.2)$ \\
\hline $\begin{array}{l}\text { General Certificate of } \\
\text { Secondary Education } \\
\left(10^{\text {th }} \text { Grade }\right)\end{array}$ & $38(27)$ & 350 & $97(27.7)$ & $89(25.4)$ & $77(22.0)$ & $59(16.9)$ & $28(8.0)$ \\
\hline $\begin{array}{l}\text { International Baccalaureate } \\
\left(12^{\text {th }} / 13^{\text {th }} \text { Grade }\right)\end{array}$ & $43(26)$ & 1,100 & $201(18.3)$ & $301(27.4)$ & $233(21.2)$ & $262(23.8)$ & $103(9.4)$ \\
\hline Other certification & $18(14)$ & 5 & $2(40.0)$ & $3(60.0)$ & $0(0.0)$ & $0(0.0)$ & $0(0.0)$ \\
\hline None & $22(18)$ & 8 & $3(37.5)$ & $3(37.5)$ & $2(25.0)$ & $0(0.0)$ & $0(0.0)$ \\
\hline Education (occupational) & & & & & & & \\
\hline Vocational School/Apprenticeship & $33(26)$ & 724 & $247(34.1)$ & $191(26.4)$ & $147(20.3)$ & $98(13.5)$ & $41(5.7)$ \\
\hline Technical School/Master craftsman & $38(25)$ & 360 & $76(21.1)$ & $114(31.7)$ & $81(22.5)$ & $69(19.2)$ & $20(5.6)$ \\
\hline University of Applied Sciences & $44(27)$ & 874 & $166(19.0)$ & $223(25.5)$ & $177(20.3)$ & $217(24.8)$ & $91(10.4)$ \\
\hline Other qualification & $39(28)$ & 41 & $10(24.4)$ & $13(31.7)$ & $5(12.2)$ & $10(24.4)$ & $3(7.3)$ \\
\hline None & $40(28)$ & 52 & $14(26.9)$ & $12(23.1)$ & $12(23.1)$ & $7(13.5)$ & $7(13.5)$ \\
\hline SES & & & & & & & \\
\hline Low $(<7.8)$ & $32(25)$ & 98 & $34(34.7)$ & $26(26.5)$ & $21(21.4)$ & $14(14.3)$ & $3(3.1)$ \\
\hline Intermediate (7.8-14) & $34(26)$ & 838 & $275(32.8)$ & $227(27.1)$ & $165(19.7)$ & $120(14.3)$ & $51(6.1)$ \\
\hline High $(>14)$ & $43(26)$ & 1,120 & $205(18.3)$ & $302(27.0)$ & $238(21.3)$ & $267(23.8)$ & $108(9.6)$ \\
\hline Marital Status & & & & & & & \\
\hline Married & $39(26)$ & 1,543 & $381(24.7)$ & $429(27.8)$ & $319(20.7)$ & $295(19.1)$ & $119(7.7)$ \\
\hline Registered partners & $35(17)$ & 3 & $0(0.0)$ & $2(66.7)$ & $1(33.3)$ & $0(0.0)$ & $0(0.0)$ \\
\hline Divorced & $38(28)$ & 140 & $42(30.0)$ & $33(23.6)$ & $27(19.3)$ & $24(17.1)$ & $14(10.0)$ \\
\hline Separated & $46(26)$ & 38 & $6(15.8)$ & $8(21.1)$ & $9(23.7)$ & $11(28.9)$ & $4(10.5)$ \\
\hline Widow(er) & $32(26)$ & 16 & $5(31.3)$ & $6(37.5)$ & $0(0.0)$ & $4(25.0)$ & $1(6.3)$ \\
\hline Single, never married & $40(27)$ & 316 & $80(25.3)$ & $77(24.4)$ & $68(21.5)$ & $67(21.2)$ & $24(7.6)$ \\
\hline Biological children & & & & & & & \\
\hline 0 & $38(27)$ & 549 & $150(27.3)$ & $145(26.4)$ & $114(20.8)$ & $98(17.9)$ & $42(7.7)$ \\
\hline $1-2$ & $39(26)$ & 1,227 & $295(24.0)$ & $342(27.9)$ & $251(20.5)$ & $241(19.6)$ & $98(8.0)$ \\
\hline $3+$ & $40(27)$ & 280 & $69(24.6)$ & $68(24.3)$ & $59(21.1)$ & $62(22.1)$ & $22(7.9)$ \\
\hline Children $<18 y$ at home & & & & & & & \\
\hline 0 & $39(27)$ & 1,932 & $492(25.5)$ & $522(27.0)$ & $395(20.4)$ & $372(19.3)$ & $151(7.8)$ \\
\hline $1-2-$ & $45(26)$ & 102 & $15(14.7)$ & $28(27.5)$ & $25(24.5)$ & $23(22.5)$ & $11(10.8)$ \\
\hline $3+$ & $35(25)$ & 22 & $7(31.8)$ & $5(22.7)$ & $4(18.2)$ & $6(27.3)$ & $0(0.0)$ \\
\hline People in household & & & & & & & \\
\hline $1-2$ & $36(27)$ & 918 & $280(30.5)$ & $233(25.4)$ & 171 (18.6) & 173 (18.8) & $61(6.6)$ \\
\hline $3-4$ & $42(26)$ & 972 & $198(20.4)$ & $272(28.0)$ & $217(22.3)$ & $195(20.1)$ & $90(9.3)$ \\
\hline $5+$ & $40(26)$ & 154 & $32(20.8)$ & $46(29.9)$ & $34(22.1)$ & $32(20.8)$ & $10(6.5)$ \\
\hline
\end{tabular}


Table 1 Private life characteristics and WPC among men (Continued)

\begin{tabular}{|c|c|c|c|c|c|c|c|}
\hline \multicolumn{8}{|l|}{ Time spent caring for children } \\
\hline $0 \mathrm{~h} /$ week & $37(26)$ & 1,355 & $382(28.2)$ & $362(26.7)$ & $269(19.9)$ & $246(18.2)$ & $96(7.1)$ \\
\hline 1-3 h/week & $43(26)$ & 480 & $115(24.0)$ & $17(3.5)$ & $142(29.6)$ & $144(30.0)$ & $62(12.9)$ \\
\hline $4+$ hours/week & $36(26)$ & 65 & $16(24.6)$ & $22(33.8)$ & $12(18.5)$ & $11(16.9)$ & $4(6.2)$ \\
\hline \multicolumn{8}{|c|}{ Time spent caring for adult relatives } \\
\hline $0 \mathrm{~h} /$ week & $39(27)$ & 1,948 & $489(25.1)$ & $530(27.2)$ & $402(20.6)$ & $371(19.0)$ & $156(8.0)$ \\
\hline 1-3 h/week & $41(27)$ & 103 & $24(23.3)$ & $24(23.3)$ & $20(19.4)$ & $29(28.2)$ & $6(5.8)$ \\
\hline $4+$ hours/week & $44(25)$ & 4 & $1(25.0)$ & $1(25.0)$ & $1(25.0)$ & $1(25.0)$ & $0(0.0)$ \\
\hline \multicolumn{8}{|c|}{ Time spent on household errands } \\
\hline $0 \mathrm{~h} /$ week & $52(27)$ & 155 & $20(12.9)$ & $32(20.6)$ & $32(20.6)$ & $41(26.5)$ & $30(19.4)$ \\
\hline $1-3 \mathrm{~h} /$ week & $38(26)$ & 1,722 & $435(25.3)$ & $482(28.0)$ & $353(20.5)$ & $332(19.3)$ & $120(7.0)$ \\
\hline 4+ hours/week & $35(26)$ & 172 & $57(33.1)$ & $41(23.8)$ & $37(21.5)$ & $25(14.5)$ & $12(7.0)$ \\
\hline \multicolumn{8}{|l|}{ Time spent on hobbies } \\
\hline $0 \mathrm{~h} /$ week & $44(27)$ & 429 & $89(20.7)$ & $93(21.7)$ & $92(21.4)$ & $115(26.8)$ & $40(9.3)$ \\
\hline 1-3 h/week & $38(26)$ & 1,477 & $374(25.3)$ & $410(27.8)$ & $303(20.5)$ & $275(18.6)$ & $115(7.8)$ \\
\hline $4+$ hours/week & $30(23)$ & 146 & 49 (33.6) & $52(35.6)$ & $29(19.9)$ & $9(6.2)$ & $7(4.8)$ \\
\hline \multicolumn{8}{|l|}{ Time spent on job development } \\
\hline $0 \mathrm{~h} /$ week & $38(27)$ & 1,726 & $459(26.6)$ & $463(26.8)$ & $349(20.2)$ & $319(18.5)$ & $136(7.9)$ \\
\hline 1-3 h/week & $44(25)$ & 325 & $55(16.9)$ & $89(27.4)$ & $73(22.5)$ & $82(25.2)$ & $26(8.0)$ \\
\hline $4+$ hours/week & $35(12)$ & 4 & $0(0.0)$ & $3(75.0)$ & $1(25.0)$ & $0(0.0)$ & $0(0.0)$ \\
\hline \multicolumn{8}{|l|}{ Smoking Status } \\
\hline Never & $41(26)$ & 851 & $184(21.6)$ & $230(27.0)$ & $184(21.6)$ & $183(21.5)$ & $70(8.2)$ \\
\hline Quit 0-2 y ago & $37(26)$ & 64 & $18(28.1)$ & 12 (18.8) & 19 (29.7) & 10 (15.6) & $5(7.8)$ \\
\hline Quit 2+y ago & $37(27)$ & 661 & $191(28.9)$ & 177 (26.8) & 125 (18.9) & $120(18.2)$ & $48(7.3)$ \\
\hline Current & $38(26)$ & 478 & $121(25.3)$ & $136(28.5)$ & $96(20.1)$ & 86 (18.0) & $39(8.2)$ \\
\hline \multicolumn{8}{|l|}{ Pack-years (PY) } \\
\hline Never smoked & $41(26)$ & 851 & $184(21.6)$ & $230(27.0)$ & $184(21.6)$ & $183(21.5)$ & $70(8.2)$ \\
\hline$<20$ PY & $37(26)$ & 871 & $233(26.8)$ & $244(28.0)$ & $174(20.0)$ & 157 (18.0) & $63(7.2)$ \\
\hline 20-39 PY & $37(27)$ & 142 & $42(29.6)$ & $32(22.5)$ & $33(23.2)$ & $24(16.9)$ & $11(7.7)$ \\
\hline $40+P Y$ & $43(28)$ & 58 & $10(17.2)$ & $20(34.5)$ & $8(13.8)$ & $13(22.4)$ & $7(12.1)$ \\
\hline \multicolumn{8}{|l|}{ Alcohol intake } \\
\hline No intake & $38(26)$ & 702 & $182(25.9)$ & 187 (26.6) & $152(21.7)$ & $129(18.4)$ & $52(7.4)$ \\
\hline Intake beneath tolerable limit & $40(26)$ & 759 & $169(22.3)$ & $213(28.1)$ & $168(22.1)$ & $148(19.5)$ & $61(8.0)$ \\
\hline Intake above tolerable limit & $38(28)$ & 522 & $146(28.0)$ & $132(25.3)$ & $91(17.4)$ & $109(20.9)$ & $44(8.4)$ \\
\hline Abuse of alcohol & $39(27)$ & 71 & $16(22.5)$ & $22(31.0)$ & $13(18.3)$ & $15(21.1)$ & $5(7.0)$ \\
\hline \multicolumn{8}{|l|}{ Depression } \\
\hline No & $38(26)$ & 1944 & $506(26.0)$ & $536(27.6)$ & $399(20.5)$ & $368(18.9)$ & $135(6.9)$ \\
\hline Yes & $58(26)$ & 110 & $8(7.3)$ & $18(16.4)$ & $24(21.8)$ & $33(30.0)$ & $27(24.5)$ \\
\hline
\end{tabular}

TOAM limits for men: beneath tolerable limit < =20 g/day; above tolerable limit $>20-60 \mathrm{~g} /$ day; abuse $>60 \mathrm{~g} / \mathrm{day}$

and male self-employed academics (12.3\%) often had a very high WPC. Furthermore, men who worked as a manager $(11.0 \%)$ and those with full-time employment (8.1\%) frequently had a very high WPC.

Even though some differences were found, distributions were largely the same for women (Table 4). Also women with highly complex tasks $(10.3 \%)$ often reported a very high WPC. Moreover, nearly a third of all women in full-time employment had a high $(18.8 \%)$ or very high WPC (10.7\%). Just as in men, doing night shifts (16.4\%) and working as manager (12.2\%) was frequently associated with a very high WPC. Similarly 
Table 2 Private life characteristics and WPC among women

\begin{tabular}{|c|c|c|c|c|c|c|c|}
\hline & \multirow[b]{2}{*}{ Mean (SD) of WPC } & \multirow[b]{2}{*}{$n$} & \multicolumn{5}{|l|}{ WPC n (\%) } \\
\hline & & & $\begin{array}{l}\text { Very low } \\
(<20 \%)\end{array}$ & $\begin{array}{l}\text { Low } \\
(20-39 \%)\end{array}$ & $\begin{array}{l}\text { Moderate } \\
(40-59 \%)\end{array}$ & $\begin{array}{l}\text { High } \\
(60-79 \%)\end{array}$ & $\begin{array}{l}\text { Very high } \\
(>=80 \%)\end{array}$ \\
\hline & & 1,653 & $562(34.0)$ & $387(23.4)$ & $323(19.5)$ & $263(15.9)$ & $118(7.1)$ \\
\hline \multicolumn{8}{|l|}{ Alter } \\
\hline $35-44$ & $36(28)$ & 608 & $197(32.4)$ & $148(24.3)$ & $104(17.1)$ & 109 (17.9) & $50(8.2)$ \\
\hline $45-54$ & $35(27)$ & 689 & $227(32.9)$ & $164(23.8)$ & $148(21.5)$ & $105(15.2)$ & $45(6.5)$ \\
\hline $55-64$ & $32(27)$ & 356 & $138(38.8)$ & $75(21.1)$ & $71(19.9)$ & 49 (13.8) & $23(6.5)$ \\
\hline \multicolumn{8}{|l|}{ Education (school) } \\
\hline $\begin{array}{l}\text { Certificate of Secondary } \\
\text { Education (9th Grade) }\end{array}$ & $30(28)$ & 367 & $160(43.6)$ & $73(19.9)$ & $63(17.2)$ & 49 (13.4) & $22(6.0)$ \\
\hline $\begin{array}{l}\text { General Certificate of Secondary } \\
\text { Education (10th Grade) }\end{array}$ & $30(26)$ & 506 & $207(40.9)$ & $117(23.1)$ & $93(18.4)$ & $63(12.5)$ & $26(5.1)$ \\
\hline $\begin{array}{l}\text { International Baccalaureate } \\
\text { (12th/13th Grade) }\end{array}$ & $40(27)$ & 760 & $188(24.7)$ & $194(25.5)$ & $163(21.4)$ & $146(19.2)$ & $69(9.1)$ \\
\hline Other certification & $39(27)$ & 15 & $5(33.3)$ & $3(20.0)$ & $4(26.7)$ & $2(13.3)$ & $1(6.7)$ \\
\hline None & $40(37)$ & 5 & $2(40.0)$ & $0(0.0)$ & $0(0.0)$ & $3(60.0)$ & $0(0.0)$ \\
\hline \multicolumn{8}{|l|}{ Education (occupational) } \\
\hline Vocational School/ Apprenticeship & $30(27)$ & 791 & $332(42.0)$ & $175(22.1)$ & $138(17.4)$ & $103(13.0)$ & $43(5.4)$ \\
\hline Technical School/Master Craftsman & $35(26)$ & 229 & $72(31.4)$ & $56(24.5)$ & $53(23.1)$ & $35(15.3)$ & $13(5.7)$ \\
\hline University of Applied Sciences & $41(27)$ & 534 & $120(22.5)$ & $133(24.9)$ & $117(21.9)$ & $113(21.2)$ & $51(9.6)$ \\
\hline Other qualification & $39(27)$ & 33 & $7(21.2)$ & $11(33.3)$ & $8(24.2)$ & $4(12.1)$ & $3(9.1)$ \\
\hline None & $30(32)$ & 66 & $31(47.0)$ & $12(18.2)$ & $7(10.6)$ & $8(12.1)$ & $8(12.1)$ \\
\hline \multicolumn{8}{|l|}{ SES } \\
\hline Low $(<7.8)$ & $25(27)$ & 61 & $31(50.8)$ & $12(19.7)$ & $10(16.4)$ & $4(6.6)$ & $4(6.6)$ \\
\hline Intermediate (7.8-14) & $31(27)$ & 936 & $379(40.5)$ & $206(22.0)$ & $171(18.3)$ & $124(13.2)$ & $56(6.0)$ \\
\hline High (>14) & $41(27)$ & 521 & $152(29.2)$ & $169(32.4)$ & $142(27.3)$ & $135(25.9)$ & $58(11.1)$ \\
\hline \multicolumn{8}{|l|}{ Marital Status } \\
\hline Married & $33(27)$ & 1,101 & $391(35.5)$ & $269(24.4)$ & $213(19.3)$ & $154(14.0)$ & $74(6.7)$ \\
\hline Registered partners & $44(39)$ & 4 & $1(25.0)$ & $1(25.0)$ & $1(25.0)$ & $0(0.0)$ & $1(25.0)$ \\
\hline Divorced & $36(29)$ & 181 & $65(35.9)$ & $34(18.8)$ & $36(19.9)$ & $32(17.7)$ & $14(7.7)$ \\
\hline Separated & $46(27)$ & 43 & $7(16.3)$ & $11(25.6)$ & $9(20.9)$ & $10(23.3)$ & $6(14.0)$ \\
\hline Widow(er) & $24(27)$ & 44 & $27(61.4)$ & $4(9.1)$ & $4(9.1)$ & $8(18.2)$ & $1(2.3)$ \\
\hline Single, never married & $39(27)$ & 280 & $71(25.4)$ & $68(24.3)$ & $60(21.4)$ & $59(21.1)$ & $22(7.9)$ \\
\hline \multicolumn{8}{|l|}{ Biological children } \\
\hline 0 & $39(28)$ & 492 & $137(27.8)$ & $112(22.8)$ & $108(22.0)$ & $86(17.5)$ & $49(10.0)$ \\
\hline $1-2$ & $32(27)$ & 959 & $357(37.2)$ & $235(24.5)$ & $171(17.8)$ & $139(14.5)$ & $57(5.9)$ \\
\hline $3+$ & $35(27)$ & 202 & $68(33.7)$ & 40 (19.8) & $44(21.8)$ & 38 (18.8) & $12(5.9)$ \\
\hline \multicolumn{8}{|l|}{ Children < 18y at home } \\
\hline 0 & $34(27)$ & 1,578 & $535(33.9)$ & $374(23.7)$ & 307 (19.5) & $250(15.8)$ & $112(7.1)$ \\
\hline $1-2$ & $37(26)$ & 66 & $24(36.4)$ & $10(15.2)$ & $14(21.2)$ & $12(18.2)$ & $6(9.1)$ \\
\hline $3+$ & $27(21)$ & 9 & $3(33.3)$ & $3(33.3)$ & $2(22.2)$ & $1(11.1)$ & $0(0.0)$ \\
\hline \multicolumn{8}{|l|}{ People in household } \\
\hline $1-2$ & $35(28)$ & 910 & $308(33.8)$ & $203(22.3)$ & $180(19.8)$ & $142(15.6)$ & $77(8.5)$ \\
\hline $3-4$ & $33(26)$ & 637 & $223(35.0)$ & $165(25.9)$ & $115(18.1)$ & $99(15.5)$ & $35(5.5)$ \\
\hline $5+$ & $37(26)$ & 90 & $27(30.0)$ & $17(18.9)$ & $24(26.7)$ & $18(20.0)$ & $4(4.4)$ \\
\hline
\end{tabular}


Table 2 Private life characteristics and WPC among women (Continued)

\begin{tabular}{|c|c|c|c|c|c|c|c|}
\hline \multicolumn{8}{|l|}{ Time spent caring for children } \\
\hline 0 h/week & $34(28)$ & 1,109 & $385(34.7)$ & $257(23.2)$ & $221(19.9)$ & $160(14.4)$ & $86(7.8)$ \\
\hline 1-3 h/week & $35(27)$ & 346 & $121(35.0)$ & $77(22.3)$ & $58(16.8)$ & $67(19.4)$ & $23(6.6)$ \\
\hline $4+$ hours/week & $35(25)$ & 195 & $56(28.7)$ & $52(26.7)$ & $43(22.1)$ & $36(18.5)$ & $8(4.1)$ \\
\hline \multicolumn{8}{|c|}{ Time spent caring for adult relatives } \\
\hline $0 \mathrm{~h} /$ week & $34(27)$ & 1,483 & $502(33.9)$ & $357(24.1)$ & $292(19.7)$ & $229(15.4)$ & $103(6.9)$ \\
\hline 1-3 h/week & $38(29)$ & 154 & $54(35.1)$ & $26(16.9)$ & $29(18.8)$ & $31(20.1)$ & $14(9.1)$ \\
\hline $4+$ hours/week & $34(32)$ & 14 & $6(42.9)$ & $3(21.4)$ & $1(7.1)$ & $3(21.4)$ & $1(7.1)$ \\
\hline \multicolumn{8}{|c|}{ Time spent on household errands } \\
\hline $0 \mathrm{~h} /$ week & $31(31)$ & 18 & $8(44.4)$ & $3(16.7)$ & $3(16.7)$ & $3(16.7)$ & $1(5.6)$ \\
\hline 1-3 h/week & $36(27)$ & 1,154 & $368(31.9)$ & $286(24.8)$ & $225(19.5)$ & $181(15.7)$ & $94(8.1)$ \\
\hline 4+ hours/week & $32(27)$ & 475 & $185(38.9)$ & $97(20.4)$ & 93 (19.6) & $78(16.4)$ & $22(4.6)$ \\
\hline \multicolumn{8}{|l|}{ Time spent on hobbies } \\
\hline $0 \mathrm{~h} /$ week & $41(29)$ & 300 & $81(27.0)$ & $64(21.3)$ & $55(18.3)$ & $67(22.3)$ & $33(11.0)$ \\
\hline 1-3 h/week & $34(27)$ & 1,241 & $429(34.6)$ & $295(23.8)$ & $247(19.9)$ & $188(15.1)$ & $82(6.6)$ \\
\hline $4+$ hours/week & $25(24)$ & 108 & $49(45.4)$ & $27(25.0)$ & $21(19.4)$ & $8(7.4)$ & $3(2.8)$ \\
\hline \multicolumn{8}{|l|}{ Time spent on job development } \\
\hline $0 \mathrm{~h} /$ week & $34(27)$ & 1,418 & $501(35.3)$ & $321(22.6)$ & $279(19.7)$ & $220(15.5)$ & $97(6.8)$ \\
\hline 1-3 h/week & $39(27)$ & 230 & $59(25.7)$ & $65(28.3)$ & $43(18.7)$ & $43(18.7)$ & $20(8.7)$ \\
\hline $4+$ hours/week & $18(10)$ & 3 & $2(66.7)$ & $1(33.3)$ & $0(0.0)$ & $0(0.0)$ & $0(0.0)$ \\
\hline \multicolumn{8}{|l|}{ Smoking Status } \\
\hline Never & $35(28)$ & 771 & $267(34.6)$ & $186(24.1)$ & $130(16.9)$ & $125(16.2)$ & $63(8.2)$ \\
\hline Quit 0-2y ago & $32(28)$ & 35 & $11(31.4)$ & $11(31.4)$ & $5(14.3)$ & $6(17.1)$ & $2(5.7)$ \\
\hline Quit 2+y ago & $35(27)$ & 463 & $148(32.0)$ & $106(22.9)$ & $114(24.6)$ & $66(14.3)$ & $29(6.3)$ \\
\hline Current & $34(27)$ & 383 & $136(35.5)$ & $83(21.7)$ & 74 (19.3) & $66(17.2)$ & $24(6.3)$ \\
\hline \multicolumn{8}{|l|}{ Pack-years } \\
\hline Never smoked & $35(28)$ & 771 & $267(34.6)$ & $186(24.1)$ & 130 (16.9) & $125(16.2)$ & $63(8.2)$ \\
\hline$<20$ PY & $34(27)$ & 720 & $243(33.8)$ & $162(22.5)$ & $160(22.2)$ & $111(15.4)$ & $44(6.1)$ \\
\hline 20-39 PY & $34(26)$ & 102 & $33(32.4)$ & $25(24.5)$ & $23(22.5)$ & 16 (15.7) & $5(4.9)$ \\
\hline $40+P Y$ & $40(34)$ & 20 & $8(40.0)$ & $2(10.0)$ & $2(10.0)$ & $6(30.0)$ & $2(10.0)$ \\
\hline \multicolumn{8}{|l|}{ Alcohol intake } \\
\hline No intake & $34(27)$ & 810 & $280(34.6)$ & $190(23.5)$ & 158 (19.5) & 126 (15.6) & $56(6.9)$ \\
\hline Intake beneath tolerable limit & $35(28)$ & 440 & $152(34.5)$ & $92(20.9)$ & $84(19.1)$ & $76(17.3)$ & $36(8.2)$ \\
\hline Intake above tolerable limit & $34(26)$ & 370 & $120(32.4)$ & $98(26.5)$ & $73(19.7)$ & $56(15.1)$ & $23(6.2)$ \\
\hline Abuse of alcohol & $37(29)$ & 33 & $10(30.3)$ & $7(21.2)$ & $8(24.2)$ & $5(15.2)$ & $3(9.1)$ \\
\hline \multicolumn{8}{|l|}{ Depression } \\
\hline No & $32(26)$ & 1,487 & $541(36.4)$ & $360(24.2)$ & $288(19.4)$ & $215(14.5)$ & $83(5.6)$ \\
\hline Yes & $55(28)$ & 162 & $21(13.0)$ & $25(15.4)$ & $33(20.4)$ & $48(29.6)$ & $35(21.6)$ \\
\hline
\end{tabular}

${ }^{\mathrm{b}}$ TOAM limits for women: beneath tolerable limit $<=10 \mathrm{~g} /$ day; above tolerable limit $>10-40 \mathrm{~g} /$ day; abuse $>40 \mathrm{~g} /$ day

to men, $14.0 \%$ of the female self-employed academics reported a very high WPC.

\section{Psychosocial working conditions}

Generally, among both women and men, adverse psychosocial working conditions were associated with a higher WPC and favourable psychosocial working conditions with a lower WPC (Tables 5 and 6). In particular, high scores on "quantitative demands","emotional demands", "demands for hiding emotions", (low)"work ability" and "burnout" were related to a high level of WPC in both women and men. In 
Table 3 Occupational characteristics and WPC among men

\begin{tabular}{|c|c|c|c|c|c|c|c|}
\hline & & & WPC n(\%) & & & & \\
\hline & Mean (SD) of WPC & $n$ & $\begin{array}{l}\text { Very low } \\
(<20 \%)\end{array}$ & $\begin{array}{l}\text { Low } \\
(20-39 \%)\end{array}$ & $\begin{array}{l}\text { Moderate } \\
(40-59 \%)\end{array}$ & $\begin{array}{l}\text { High } \\
(60-79 \%)\end{array}$ & $\begin{array}{l}\text { Very high } \\
(>=80 \%)\end{array}$ \\
\hline Form of Employment & & & & & & & \\
\hline Full-time & $39(27)$ & 1,990 & $490(24.6)$ & $528(26.5)$ & $417(21.0)$ & 394 (19.8) & $161(8.1)$ \\
\hline Part-time & $26(22)$ & 66 & $24(36.4)$ & $27(40.9)$ & $7(10.6)$ & $7(10.6)$ & $1(1.5)$ \\
\hline Time spent at work & & & & & & & \\
\hline$<40$ [h/week] & $30(25)$ & 679 & $241(35.5)$ & 209 (30.8) & $117(17.2)$ & 79 (11.6) & $33(4.9)$ \\
\hline 40+ [h/week] & $43(26)$ & 1,312 & $263(20.0)$ & $334(25.5)$ & $288(22.0)$ & $305(23.2)$ & $122(9.3)$ \\
\hline Night shift & & & & & & & \\
\hline No & $37(26)$ & 1,647 & $437(26.5)$ & $466(28.3)$ & $332(20.2)$ & 304 (18.5) & $108(6.6)$ \\
\hline Yes & $47(27)$ & 372 & $65(17.5)$ & $78(21.0)$ & $86(23.1)$ & $94(25.3)$ & 49 (13.2) \\
\hline Night Work & & & & & & & \\
\hline 0-6 [days/month] & $38(26)$ & 1,893 & $481(25.4)$ & $519(27.4)$ & $393(20.8)$ & $360(19.0)$ & $140(7.4)$ \\
\hline 7+ [days/month] & $48(27)$ & 126 & $21(16.7)$ & $25(19.8)$ & 25 (19.8) & $38(30.2)$ & $17(13.5)$ \\
\hline Job complexity level & & & & & & & \\
\hline Low & $29(28)$ & 23 & $10(43.5)$ & $5(21.7)$ & $5(21.7)$ & $1(4.3)$ & $2(8.7)$ \\
\hline Medium & $33(26)$ & 716 & $241(33.7)$ & $195(27.2)$ & $144(20.1)$ & $104(14.5)$ & $32(4.5)$ \\
\hline Complex & $40(27)$ & 488 & $116(23.8)$ & $131(26.8)$ & $93(19.1)$ & $105(21.5)$ & $43(8.8)$ \\
\hline Very complex & $44(26)$ & 792 & $135(17.0)$ & $213(26.9)$ & $176(22.2)$ & $188(23.7)$ & $80(10.1)$ \\
\hline Management & & & & & & & \\
\hline No & $37(26)$ & 1,600 & $448(28.0)$ & $423(26.4)$ & $324(20.3)$ & $293(18.3)$ & $112(7.0)$ \\
\hline Yes & $45(26)$ & 456 & $66(14.5)$ & $132(28.9)$ & $100(21.9)$ & $108(23.7)$ & $50(11.0)$ \\
\hline Position & & & & & & & \\
\hline Worker & $30(25)$ & 279 & $104(37.3)$ & $73(26.2)$ & $53(19.0)$ & $37(13.3)$ & $12(4.3)$ \\
\hline Employee & $39(26)$ & 1,190 & $286(24.0)$ & $326(27.4)$ & $262(22.0)$ & $216(18.2)$ & $100(8.4)$ \\
\hline $\begin{array}{l}\text { Government officials, judges, } \\
\text { military employees }\end{array}$ & $39(26)$ & 166 & 39 (23.5) & $46(27.7)$ & $34(20.5)$ & $37(22.3)$ & $10(6.0)$ \\
\hline $\begin{array}{l}\text { Self-employed/cooperative } \\
\text { agriculturalist }\end{array}$ & $47(25)$ & 43 & $7(16.3)$ & $9(20.9)$ & $10(23.3)$ & $15(34.9)$ & $2(4.7)$ \\
\hline $\begin{array}{l}\text { Self-employed in trade, } \\
\text { commerce, craftwork, industry, } \\
\text { service (also freelancers) }\end{array}$ & $43(27)$ & 312 & $63(20.2)$ & $89(28.5)$ & $54(17.3)$ & $77(24.7)$ & $29(9.3)$ \\
\hline $\begin{array}{l}\text { Academic self-employed } \\
\text { profession (physician, } \\
\text { attorney, tax consultant) }\end{array}$ & $45(29)$ & 65 & $15(23.1)$ & $12(18.5)$ & $11(16.9)$ & $19(29.2)$ & $8(12.3)$ \\
\hline Student/trainee & - & - & - & - & - & - & - \\
\hline Caretaker for relatives & - & - & - & - & - & - & - \\
\hline
\end{tabular}

women, "role conflicts" and "cognitive stress" played an additional important role. On the other hand, "degree of freedom at work", "quality of leadership", "social support", "job satisfaction" and "life satisfaction" were associated with a low WPC in both women and men.

Still, some of the favourable psychosocial working conditions, i.e. "possibilities for development" (particularly in women) and "workplace commitment", were tendentially positively associated with WPC.

\section{Multivariable results}

Table 7 shows the results of the Poisson regression analyses for women and men separately as well as for all subjects together in one analysis. In the regression models, both socio-demographic as well as occupational characteristics were included. Stepwise selection led to somewhat different models for the respective analyses with a little different set of variables (Table 7). For men, the WPC risk (as expressed by the prevalence ratio) was increased when they spent much time at 
Table 4 Occupational characteristics and WPC among women

\begin{tabular}{|c|c|c|c|c|c|c|c|}
\hline & & & WPC n(\%) & & & & \\
\hline & Mean (SD) of WPC & $n$ & $\begin{array}{l}\text { Very low } \\
(<20 \%)\end{array}$ & $\begin{array}{l}\text { Low } \\
(20-39 \%)\end{array}$ & $\begin{array}{l}\text { Moderate } \\
(40-59 \%)\end{array}$ & $\begin{array}{l}\text { High } \\
(60-79 \%)\end{array}$ & $\begin{array}{l}\text { Very high } \\
(>=80 \%)\end{array}$ \\
\hline Form of Employment & & & & & & & \\
\hline Full-time & $41(28)$ & 919 & $236(25.7)$ & $203(22.1)$ & $209(22.7)$ & $173(18.8)$ & $98(10.7)$ \\
\hline Part-time & $27(24)$ & 734 & $326(44.4)$ & $184(25.1)$ & $114(15.5)$ & $90(12.3)$ & $20(2.7)$ \\
\hline Time spent at work & & & & & & & \\
\hline$<40$ [h/week] & $31(27)$ & 1226 & $468(38.2)$ & 301 (24.6) & $210(17.1)$ & $183(14.9)$ & $64(5.2)$ \\
\hline $40+[h /$ week $]$ & $44(28)$ & 405 & $89(22.0)$ & 80 (19.8) & $108(26.7)$ & $76(18.8)$ & $52(12.8)$ \\
\hline Night shift & & & & & & & \\
\hline No & $33(27)$ & 1508 & $542(35.9)$ & $358(23.7)$ & $283(18.8)$ & $228(15.1)$ & $97(6.4)$ \\
\hline Yes & $51(26)$ & 128 & $15(11.7)$ & $24(18.8)$ & $38(29.7)$ & $30(23.4)$ & $21(16.4)$ \\
\hline Night Work & & & & & & & \\
\hline 0-6 [days/month] & $34(27)$ & 1603 & $554(34.6)$ & $374(23.3)$ & 314 (19.6) & $251(15.7)$ & $110(6.9)$ \\
\hline $7+$ [days/month] & $54(27)$ & 33 & $3(9.1)$ & $8(24.2)$ & $7(21.2)$ & $7(21.2)$ & $8(24.2)$ \\
\hline Job complexity level & & & & & & & \\
\hline Low & $27(30)$ & 82 & $41(50.0)$ & $16(19.5)$ & $10(12.2)$ & $8(9.8)$ & $7(8.5)$ \\
\hline Medium & $30(26)$ & 861 & $357(41.5)$ & $200(23.2)$ & $154(17.9)$ & $103(12.0)$ & $47(5.5)$ \\
\hline Complex & $39(28)$ & 303 & $84(27.7)$ & $65(21.5)$ & $66(21.8)$ & $64(21.1)$ & $24(7.9)$ \\
\hline Very complex & $43(26)$ & 390 & $75(19.2)$ & $101(25.9)$ & $91(23.3)$ & $83(21.3)$ & $40(10.3)$ \\
\hline Management & & & & & & & \\
\hline No & $33(27)$ & 1506 & $536(35.6)$ & $358(23.8)$ & $289(19.2)$ & $223(14.8)$ & $100(6.6)$ \\
\hline Yes & $47(27)$ & 147 & $26(17.7)$ & $29(19.7)$ & $34(23.1)$ & $40(27.2)$ & $18(12.2)$ \\
\hline Position & & & & & & & \\
\hline Worker & $26(30)$ & 52 & $27(51.9)$ & $10(19.2)$ & $7(13.5)$ & $3(5.8)$ & $5(9.6)$ \\
\hline Employee & $33(27)$ & 1254 & $450(35.9)$ & $294(23.4)$ & $235(18.7)$ & 195 (15.6) & $80(6.4)$ \\
\hline $\begin{array}{l}\text { Government officials, judges, } \\
\text { military employees }\end{array}$ & $43(26)$ & 135 & $25(18.5)$ & $34(25.2)$ & 35 (25.9) & $29(21.5)$ & $12(8.9)$ \\
\hline $\begin{array}{l}\text { Self-employed/cooperative } \\
\text { agriculturalist }\end{array}$ & $49(26)$ & 10 & $2(20.0)$ & $1(10.0)$ & $2(20.0)$ & $4(40.0)$ & $1(10.0)$ \\
\hline $\begin{array}{l}\text { Self-employed in trade, commerce, } \\
\text { craftwork, industry, service } \\
\text { (also freelancers) }\end{array}$ & $37(28)$ & 149 & $45(30.2)$ & $36(24.2)$ & $30(20.1)$ & $25(16.8)$ & $13(8.7)$ \\
\hline $\begin{array}{l}\text { Academic self-employed profession } \\
\text { (physician, attorney, tax consultant) }\end{array}$ & $43(28)$ & 43 & $10(23.3)$ & $11(25.6)$ & $10(23.3)$ & $6(14.0)$ & $6(14.0)$ \\
\hline Student/trainee & $55(18)$ & 3 & $0(0.0)$ & $0(0.0)$ & $2(66.7)$ & $1(33.3)$ & $0(0.0)$ \\
\hline Caretaker for relatives & $41(41)$ & 4 & $1(25.0)$ & $1(25.0)$ & $1(25.0)$ & $0(0.0)$ & $1(25.0)$ \\
\hline
\end{tabular}

work (more than $40 \mathrm{~h}$ a week) and spent time for caring for adult relatives (PR 1.60, $95 \%$ CI 1.29-1.99 and PR 1.24, 95 \% CI 1.07-1.43 respectively). Smoking and spending time on household errands was associated with a lower WPC risk (PR 0.72, 95 \% CI 0.53-0.97 and PR 0.91, 95 \% CI 0.83-0.99 respectively). For women, particularly depression was associated with an elevated risk for a WPC (PR 1.99, 95 \% CI 1.53-2.59). Opposed to men, further explanatory variables for an increased WPC risk in women were a high amount of night shifts (PR 1.92, 95 \% CI 1.25-2.93), being divorced (PR 1.41,
$95 \%$ CI 1.07-1.86) or separated (PR 1.69, $95 \%$ CI 1.06-2.71) and holding a management position (PR $1.35,95 \%$ CI 1.03-1.76). The regression model including both men and women showed that, contrary to the descriptive results (Tables 1 and 2), in the adjusted multivariable model women had a higher risk for WPC compared to men (Table 7). Further, according to the overall model the WPC risk was reduced for persons with part-time work (PR 0.50, $95 \%$ CI 0.39-0.64), for older persons (PR 0.83, $95 \%$ CI 0.76-0.91) and for persons not spending time on hobbies (PR 0.88, $95 \% \mathrm{CI}$ 
Table 5 Psychosocial working conditions as overall mean (SD) and in relation to WPC category, men

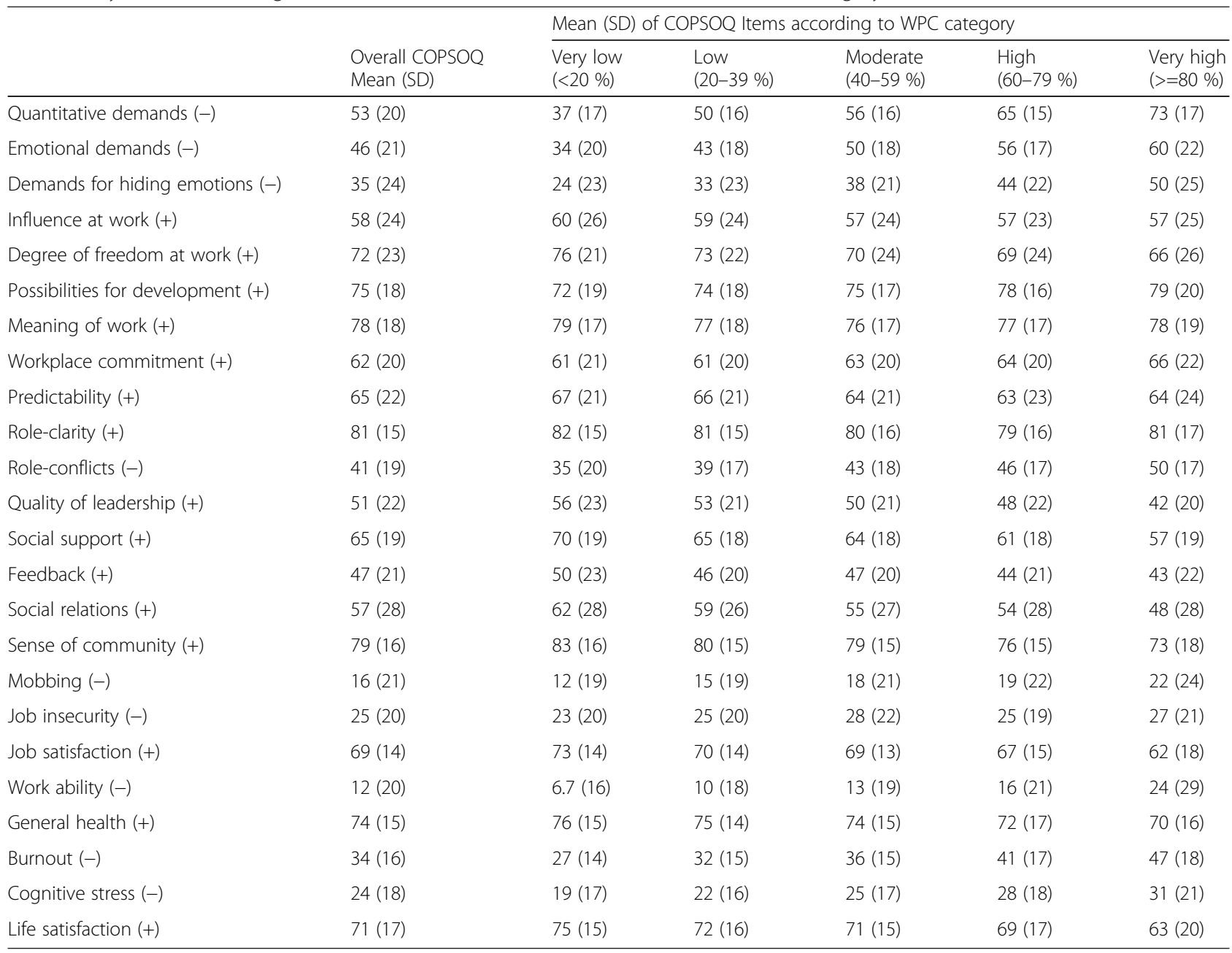

0.82-0.93). On the other hand, there were a number of variables that were associated with an elevated risk for a WPC: both a higher SES (PR 1.08, 95 \% CI 1.06-1.10) and caring for an adult relative (PR 1.15, 95 \% CI 1.061.24) were related to a WPC. Also depression (PR 1.55, $95 \%$ CI 1.28-1.89) and being separated (PR 1.53, $95 \%$ CI 1.05-2.22) from the partner was associated with an elevated WPC. With regard to occupational characteristics, working night shifts (PR 1.58, 95 \% CI 1.331.88), time spent at work (PR 1.34, $95 \%$ CI 1.15-1.55) and working as manager (PR 1.22, $95 \%$ CI 1.05-1.42) were important explanatory variables for WPC in the overall model.

Finally, the results of the sensitivity analysis (see Additional file 2) showed that the risk of a WPC was increased by $3 \%$ with each working hour per week (Overall model: PR 1.03, 95 \% CI 1.03-1.04; Men: PR 1.03, 95 \% CI 1.03-1.04, Women: PR 1.03, 95 \% CI 1.02-1.04). Also here, stepwise selection led to somewhat different models with a slightly different set of variables.

\section{Discussion}

With the changing working conditions over the last decades and the resulting psychological stress, WPC is no longer a rarity but constitutes a societal problem [10]. This is reflected in the high prevalence in the present study $(27.4 \%$ of the men and $23.0 \%$ of the women reported a high or very high WPC). In a Swiss, nationally representative study from 2009 the prevalence was "merely" $12.5 \%$ [9]. However, in that study, the researchers used another measurement instrument. This instrument comprised only two items and had a rather low reliability $(\alpha=0.53)$, while our WPC measure had an excellent reliability $(\alpha=0.91)$. Also, the Swiss study covered the age span 20-64 years, whereas the subjects in our sample were 35-64 years old. Possibly, the younger subjects in the Swiss study had fewer private obligations yet, which might have protected against a WPC.

We found very interesting results regarding sex differences. According to the descriptive results, it seemed like men had a higher risk for having a WPC. The 
Table 6 Psychosocial working conditions as overall mean (SD) and in relation to WPC category, women

\begin{tabular}{|c|c|c|c|c|c|c|}
\hline & & Mean (SD & DPSOQ Items & ding to WP & jory & \\
\hline & $\begin{array}{l}\text { Overall COPSOQ } \\
\text { Mean (SD) }\end{array}$ & $\begin{array}{l}\text { Very low } \\
(<20 \%)\end{array}$ & $\begin{array}{l}\text { Low } \\
(20-39 \%)\end{array}$ & $\begin{array}{l}\text { Moderate } \\
(40-59 \%)\end{array}$ & $\begin{array}{l}\text { High } \\
(60-79 \%)\end{array}$ & $\begin{array}{l}\text { Very high } \\
(>=80 \%)\end{array}$ \\
\hline Quantitative demands (-) & $49(20)$ & $35(18)$ & $47(16)$ & $55(16)$ & $63(15)$ & $71(15)$ \\
\hline Emotional demands (-) & $47(23)$ & $32(22)$ & $46(20)$ & $54(19)$ & $59(20)$ & $69(18)$ \\
\hline Demands for hiding emotions (-) & $37(25)$ & $26(24)$ & $37(22)$ & $43(23)$ & $47(21)$ & $56(24)$ \\
\hline Influence at work (+) & $47(26)$ & $47(28)$ & $50(25)$ & $50(25)$ & $46(24)$ & $39(23)$ \\
\hline Degree of freedom at work $(+)$ & $62(26)$ & $68(24)$ & $61(27)$ & $61(25)$ & $56(29)$ & $49(27)$ \\
\hline Possibilities for development (+) & $69(20)$ & $64(21)$ & $69(19)$ & $72(19)$ & $73(19)$ & $75(18)$ \\
\hline Meaning of work (+) & $76(18)$ & $76(19)$ & $76(17)$ & $75(18)$ & $76(18)$ & $74(19)$ \\
\hline Workplace commitment (+) & $60(20)$ & $58(21)$ & $60(18)$ & $61(20)$ & $61(19)$ & $62(20)$ \\
\hline Predictability (+) & $61(21)$ & $66(21)$ & $62(19)$ & $61(22)$ & $56(21)$ & $54(23)$ \\
\hline Role-clarity (+) & $79(16)$ & $81(16)$ & $80(15)$ & $78(16)$ & $75(18)$ & $76(19)$ \\
\hline Role-conflicts (-) & $37(20)$ & $28(19)$ & $36(18)$ & $41(18)$ & $46(20)$ & $49(22)$ \\
\hline Quality of leadership (+) & $52(23)$ & $58(24)$ & $55(22)$ & $48(23)$ & $46(22)$ & $41(23)$ \\
\hline Social support (+) & $66(20)$ & $70(21)$ & $67(17)$ & $63(21)$ & $61(19)$ & $58(18)$ \\
\hline Feedback (+) & $42(21)$ & $44(23)$ & $44(20)$ & $43(22)$ & $39(19)$ & $36(21)$ \\
\hline Social relations (+) & $55(29)$ & $60(29)$ & $57(30)$ & $53(29)$ & $50(27)$ & $48(27)$ \\
\hline Sense of community (+) & $80(17)$ & $84(15)$ & $79(15)$ & $77(17)$ & $76(17)$ & $74(19)$ \\
\hline Mobbing (-) & $16(21)$ & $11(17)$ & $14(20)$ & $19(23)$ & $23(23)$ & $22(25)$ \\
\hline Job insecurity $(-)$ & $23(20)$ & $21(19)$ & $24(20)$ & $23(20)$ & $26(21)$ & $28(23)$ \\
\hline Job satisfaction (+) & $68(15)$ & $72(15)$ & $69(13)$ & $66(15)$ & $64(16)$ & $60(16)$ \\
\hline Work ability (-) & $14(21)$ & $7.8(17)$ & $11(17)$ & $17(22)$ & $19(23)$ & $32(30)$ \\
\hline General health (+) & $73(17)$ & $76(16)$ & $74(16)$ & $71(18)$ & $70(19)$ & $65(18)$ \\
\hline Burnout (-) & $42(18)$ & $34(16)$ & $39(16)$ & $45(16)$ & $52(15)$ & $61(15)$ \\
\hline Cognitive stress $(-)$ & $28(19)$ & $22(17)$ & $27(19)$ & $30(18)$ & $36(20)$ & $42(20)$ \\
\hline Life satisfaction (+) & $70(19)$ & $74(19)$ & $73(16)$ & $67(20)$ & $66(18)$ & $59(19)$ \\
\hline
\end{tabular}

multivariable results however show that after adjusting for a number of confounding variables, women were of higher risk for a WPC. Similar to our descriptive results, Hämmig et al. (2009) also found that men had more often WPC. However, they only conducted their multivariable analyses separately for women and men. Our multivariable results demonstrate that an overall analysis with an adjustment for sex might be essential, as in fact, women seemed to suffer more often from WPC. In line with this, Byron (2005) found that mothers experience more WPC than fathers. Byron hypothesizes that women might tend to take on greater responsibilities for childcare, and therefore experience more distress, when they also have to deal with a considerable workload [7]. That might be why particularly for women being divorced or separated is associated with an increased risk for a WPC. Still, more mothers than fathers keep the children after a separation [36] and being a working single mother is likely to result in role strain [7]. A traditional role allocation can also be seen with regard to household errands. Almost a fifth of all men, who did not spend any time on household errands, reported a very high WPC; and we found a negative association between WPC and time spent on household errands in the multivariable analyses. On the other hand, we found a positive association between WPC and a lot of time spent at work. Among women, such a clear trend was not discernible. The time, they spent on household errands, seemed not to be associated with their WPC and was not selected in the multivariable model for women.

There was a relative strong association between WPC and depression in our study. This is not surprising and has been found previously $[37,38]$. On the one hand depression might lead to a negative response bias, so that depressed women would be more likely to report a WPC. On the other hand it is also conceivable that a high WPC (coupled with general fatigue) results in depression [39]. In the multivariable models that were stratified by sex, depression was selected only in the model for women. Hämmig et al. (2009) also found somewhat stronger associations between 
Table 7 Prevalence ratios for WPC-score $>60 \%$, results of stepwise selection based on Poisson regression model

\begin{tabular}{|c|c|c|c|}
\hline & \multicolumn{3}{|l|}{ PR $(95 \%$ Cl) } \\
\hline & Men $(n=1,800)$ & Women $(n=1,542)$ & All $(n=3,342)$ \\
\hline Sex (Women) & & & $1.25(1.08-1.44)$ \\
\hline Age (PR per 10y increase) & $0.83(0.74-0.93)$ & $0.91(0.79-1.04)$ & $0.83(0.76-0.91)$ \\
\hline SES & $1.08(1.06-1.11)$ & $1.07(1.04-1.10)$ & $1.08(1.06-1.10)$ \\
\hline Diabetes (yes) & & $0.41(0.12-1.37)$ & \\
\hline Smoking (yes) & $0.72(0.53-0.97)$ & & $0.80(0.64-0.99)$ \\
\hline Pack-years & $1.01(1.00-1.02)$ & & $1.01(1.00-1.02)$ \\
\hline Negative affectivity & $1.08(1.06-1.09)$ & $1.06(1.04-1.08)$ & $1.07(1.05-1.08)$ \\
\hline Depression & & $1.99(1.53-2.59)$ & $1.55(1.28-1.89)$ \\
\hline Biological children (per child) & & & $1.06(0.99-1.12)$ \\
\hline $\begin{array}{l}\text { Time spent caring for adult } \\
\text { relatives [hours/week] }\end{array}$ & $1.24(1.07-1.43)$ & & $1.15(1.06-1.24)$ \\
\hline $\begin{array}{l}\text { Time spent on household } \\
\text { errands [hours/week] }\end{array}$ & $0.91(0.83-0.99)$ & & \\
\hline Time spent on hobbies [hours/week] & $0.90(0.83-0.97)$ & $0.84(0.75-0.93)$ & $0.88(0.82-0.93)$ \\
\hline Divorced & & $1.41(1.07-1.86)$ & $1.22(0.98-1.51)$ \\
\hline Separated & & $1.69(1.06-2.71)$ & $1.53(1.05-2.22)$ \\
\hline Time spent at work $(40+h)$ & $1.60(1.29-1.99)$ & & $1.34(1.15-1.55)$ \\
\hline Night shift & $1.73(1.44-2.07)$ & $1.50(1.10-2.03)$ & $1.58(1.33-1.88)$ \\
\hline Night Work (7+ days/month) & & $1.92(1.25-2.93)$ & $1.33(1.02-1.72)$ \\
\hline Part-time Employment & $0.27(0.07-1.09)$ & $0.48(0.38-0.62)$ & $0.50(0.39-0.64)$ \\
\hline Management & & $1.35(1.03-1.76)$ & $1.22(1.05-1.42)$ \\
\hline
\end{tabular}

work-life conflict and mental health impairments among women. Similarly, in a longitudinal study, work-to-family conflict was found to be more detrimental to women's satisfaction and well-being than that of men [40]. An explanation for this difference might be that a negative spillover from work to private life is more stressful for women, because the family role and private life domain is more important to the woman's self-concept and social identity [9].

For both women and men, full-time employment and many of the other psychosocial risk factors at work were associated with an increased WPC. The importance of work strain and the extent of working hours for a WPC are plausible and have been documented before [9, 17, 41]. Härma (2006) therefore argues that the reduction of overtime and long working days would act as a central factor in reducing psychosocial strain and in the prevention of adverse health effects. At the same time we also found some of the favourable psychosocial working conditions ("possibilities for development", "workplace commitment") related to WPC, indicating that demanding jobs that interfere with private life also hold positive outcomes for employees.

\section{Strengths and limitations}

In the current study, we examined a representative, population-based sample. Even though the overall GHS study was set up to look at cardio-vascular risk stratification rather than understanding occupational risk distributions, we investigated a broad variety of factors potentially associated with a WPC. In fact, we included considerably more work variables and assessed more extensively private characteristics than most of the previous studies on WPC [7, 9, 10]. Moreover, we considered the entire private life and did not restrict the assessment to family life.

The cross-sectional design constitutes the main limitation of this study. Even though theoretical frameworks concerning the temporal sequence have been suggested $[10,30]$, we do not know for sure, whether the related factors are a cause or consequence of WPC. Currently, the GHS is assessing 5-year follow-up data. These data will give the opportunity to prospectively analyse WPC and thus provide a better basis for assessing causality.

\section{Conclusions}

By affecting the individual work life, home life, and the general well-being and health, WPC may lead to detrimental effects in employees, their families, employers, and society as a whole $[10,42]$. Therefore, the high prevalence of WPC in our sample should be of concern. Among women, the risk for suffering from WPC was even higher, most likely due to multiple burdens. In Germany, many women consider it to be difficult to combine work and private life, which is inter alia 
reflected in the comparatively low work participation rate among German mothers [43]. In our view, this poses a gender equality problem and calls for (political) solutions.

\section{Additional files}

Additional file 1: STROBE checklist. (DOCX $42 \mathrm{~kb}$ )

Additional file 2: Table S1. Sensitivity analysis of Poisson regression model with working hours per week instead of overtime and part-time employment. (DOCX $16 \mathrm{~kb})$

\section{Competing interests}

Philipp S. Wild is funded by the Federal Ministry of Education and Research (BMBF 01EO1003) and he received honoraria for lectures or consulting from Boehringer Ingelheim and Bayer HealthCare, Leverkusen.

\section{Authors' contributions}

SGN drafted the manuscript, JH helped her with that. SGN, JH, AS, MN, SJ, FL, and $U L$ participated in the design of the study and the interpretation of the data. CEK, PSW, and SL have been essential for the acquisition of data. All authors have been involved in revising the manuscript critically, and read and approved the final manuscript.

\section{Acknowledgements}

The authors would like to thank Andreas Schulz of the University of Mainz for his assistance in implementing the statistical analysis.

The Gutenberg Health Study is funded through the government of Rheinland-Pfalz ("Stiftung Rheinland-Pfalz für Innovation“, contract AZ 961386261/733), the research programs "Wissenschaft Zukunft" and "Center for Translational Vascular Biology (CTVB)" of the Johannes Gutenberg-University of Mainz, and its contract with Boehringer Ingelheim and PHILIPS Medical Systems, including an unrestricted grant for the Gutenberg Health Study. The Federal Institute for Occupational Safety and Health (BAuA) funded the collection of occupational variables (including the psychosocial questionnaires) within the GHS and this analysis (BAuA research project no. F2235).

\section{Author details}

Institute and Policlinic of Occupational and Social Medicine, Faculty of Medicine, TU Dresden, Fetscherstr. 74, D-01307 Dresden, Germany. 2 Department of Psychosomatics and Health Behaviour, Norwegian Institute of Public Health, Oslo, Norway. ${ }^{3}$ FFAW, Freiburg Research Centre for Occupational Sciences, Bertoldstr. 27, D-79098 Freiburg, Germany. ${ }^{4}$ Preventive Cardiology and Preventive Medicine, Department of Medicine 2, University Medical Center Mainz, Johannes Gutenberg University Mainz, Langenbeckstr. 1, D-55131 Mainz, Germany. ${ }^{5}$ Federal Institute for Occupational Safety and Health (BAuA), Nöldnerstr. 40-42, D-10317 Berlin, Germany. ${ }^{6}$ Center for Thrombosis and Hemostasis, University Medical Center Mainz, Langenbeckstr. 1, D-55131 Mainz, Germany. ${ }^{7}$ German Center for Cardiovascular Research (DZHK), partner site RhineMain, Mainz, Langenbeckstr. 1, D-55131 Mainz, Germany. Institute of Occupational, Social and Environmental Medicine, University Medical Center, Johannes Gutenberg University Mainz, Obere Zahlbacher Straße 67, D-55131 Mainz, Germany.

Received: 15 September 2015 Accepted: 17 February 2016

Published online: 29 February 2016

\section{References}

1. Eby LT, Maher CP, Butts MM. The intersection of work and family life: the role of affect. Annu Rev Psychol. 2010;61:599-622.

2. Champoux JE. Perceptions of work and nonwork: a reexamination of the compensatory and spillover models. Work Occup. 1978;5(4):402-22.

3. Staines GL. Spillover versus compensation: a review of the literature on the relationship between work and nonwork. Human Relations. 1980;33(2):111-29.

4. Greenhaus JH, Powell GN. When work and family Are allies: a theory of work-family enrichment. Acad Manag Rev. 2006;31(1):72-92.

5. Parent-Thirion A, Vermeylen G, Van Houten G, Lyly-Yriänäinen M, Cabrita J. Fifth European Working Conditions Survey. Luxembourg: Eurofound; 2012.
6. Nübling M, Seidler A, Garthus-Niegel S, Latza U, Wagner M, Hegewald J, et al. The gutenberg health study: measuring psychosocial factors at work and predicting health and work-related outcomes with the ERI and the COPSOQ questionnaire. BMC Public Health. 2013;13:538.

7. Byron K. A meta-analytic review of work-family conflict and its antecedents. J Vocat Behav. 2005;67(2):169-98.

8. Lewis $\mathrm{S}$, Cooper $\mathrm{CL}$. The work-family research agenda in changing contexts. J Occup Health Psychol. 1999;4(4):382-93.

9. Hammig O, Gutzwiller F, Bauer G. Work-life conflict and associations with work- and nonwork-related factors and with physical and mental health outcomes: a nationally representative cross-sectional study in Switzerland. BMC Public Health. 2009;9:435.

10. Allen TD, Herst DE, Bruck CS, Sutton M. Consequences associated with work-to-family conflict: a review and agenda for future research. J Occup Health Psychol. 2000;5(2):278-308.

11. Frone MR. Work-family conflict and employee psychiatric disorders: the National Comorbidity Survey. J Appl Psychol. 2000;85(6):888-95.

12. Hammer TH, Saksvik PO, Nytro K, Torvatn H, Bayazit M. Expanding the psychosocial work environment: workplace norms and work-family conflict as correlates of stress and health. J Occup Health Psychol. 2004;9(1):83-97.

13. Judge TA, Colquitt JA. Organizational justice and stress: the mediating role of work-family conflict. J Appl Psychol. 2004;89(3):395-404.

14. Major VS, Klein KJ, Ehrhart MG. Work time, work interference with family, and psychological distress. J Appl Psychol. 2002;87(3):427-36.

15. van Rijswijk K, Bekker MH, Rutte CG, Croon MA. The relationships among part-time work, work-family interference, and well-being. J Occup Health Psychol. 2004;9(4):286-95.

16. Grzywacz JG, Bass BL. Work, family, and mental health: testing different models of work-family Fit. J Marriage Fam. 2003;65(1):248-61.

17. Jansen NWH, Kant I, Kristensen TS, Nijhuis FJN. Antecedents and consequences of work-family conflict: a prospective cohort study. J Occup Environ Med. 2003:45(5):479-91.

18. Schieman S, McBrier D, Gundy K. Home-to-work conflict, work qualities, and emotional distress. Sociol Forum. 2003:18(1):137-64.

19. Adams GA, Jex SM. Relationships between time management, control, work-family conflict, and strain. J Occup Health Psychol. 1999:4(1):72-7.

20. Burke RJ, Greenglass ER. Work-family conflict, spouse support, and nursing staff well-being during organizational restructuring. J Occup Health Psychol. 1999;4(4):327-36.

21. Frone MR, Russell M, Barnes GM. Work-family conflict, gender, and health-related outcomes: a study of employed parents in two community samples. J Occup Health Psychol. 1996;1 (1):57-69.

22. Geiger-Brown JM, Lee CJ, Trinkoff AM. The role of work schedules in occupational health and safety. In: Gatchel RJS, Schultz IZ, editors. Handbook of Occupational Health and Wellness. New York: Springer US; 2012. p. 297-322.

23. Sodan AC. Societies' different strengths in computer science education and research. Soc Behav Personal. 2004;32(1):73-94.

24. Beutel ME, Wiltink J, Till Y, Wild PS, Munzel T, Ojeda FM, et al. Type D personality as a cardiovascular risk marker in the general population: results from the Gutenberg health study. Psychother Psychosom. 2012;81(2):108-17.

25. Wild PS, Zeller T, Beutel M, Blettner M, Dugi KA, Lackner KJ, et al. Die Gutenberg Gesundheitsstudie. Bundesgesundheitsblatt. 2012;55:824-30.

26. Wild PS, Sinning CR, Roth A, Wilde S, Schnabel RB, Lubos E, et al. Distribution and categorization of left ventricular measurements in the general population: results from the population-based Gutenberg Heart Study. Circ Cardiovasc Imaging. 2010;3(5):604-13.

27. Lampert T, Kroll L: Die Messung des sozioökonomischen Status in sozialepidemiologischen Studien. In: Gesundheitliche Ungleichheit. edn. Edited by Richter M, Hurrelmann K: VS Verlag für Sozialwissenschaften; 2009: 309-334.

28. Burger MM, Mensink G. Bundesgesundheits-Survey: Alkohol. Konsumverhalten in Deutschland. Berlin: Robert Koch-Institut; 2003.

29. Manea L, Gilbody S, McMillan D. Optimal cut-off score for diagnosing depression with the Patient Health Questionnaire (PHQ-9): a meta-analysis. CMAJ. 2012:184(3):E191-6.

30. Nübling M, Stößel U, Hasselhorn H-M, Michaelis M, Hofmann F: Measuring psychological stress and strain at work: Evaluation of the COPSOQ Questionnaire in Germany. GMS Psycho-Social-Medicine 2006, 3

31. Stephens GK, Sommer SM. The measurement of work to family conflict. Educ Psychol Meas. 1996;56(3):475-86. 
32. Netemeyer RGB, Boles JS, McMurrian R. Development and validation of work-family conflict and family-work conflict scales. J Appl Psychol. 1996:81(4):400-10.

33. Carlson DS, Kacmar KM, Williams LJ. The development and validation of a multi-dimensional measure of work-family conflict. Acad Manag Proc. 1998;1998(1):A1-7.

34. Team RDC. A Language and Environment for Statistical Computing. Vienna: R Foundation for Statistical Computing; 2011.

35. Venables WN, Ripley BD. Modern Applied Statistics with S. 4th ed. New York: Springer; 2002.

36. Bundesamt S. Alleinerziehende in Deutschland. Ergebnisse des Mikrozensus 2009. Wiesbaden: Statisisches Bundesamt; 2010.

37. Frone MR, Russell M, Cooper ML. Antecedents and outcomes of work-family conflict: testing a model of the work-family interface. J Appl Psychol. 1992; 77(1):65-78.

38. Macewen KE, Barling J. Daily consequences of work interference with family and family interference with work. Work Stress. 1994;8(3):244-54.

39. Frone MR, Russell M, Cooper ML. Relation of work-family conflict to health outcomes: A four-year longitudinal study of employed parents. J Occup Organ Psychol. 1997;70(4):325-35.

40. Kinnunen U, Geurts S, Mauno S. Work-to-family conflict and its relationship with satisfaction and well-being: a one-year longitudinal study on gender differences. Work Stress. 2004;18(1):1-22.

41. Härmä M. Workhours in relation to work stress, recovery and health. Scand Work Environ Health. 2006;32(6):502-14.

42. Parasuraman SG, J.H.: The changing world of work and family. In: Integrating work and family: Challenges for a changing world. edn. Edited by Parasuraman SG, J.H. Westport, CT: Quorum Books; 1997: 3-14.

43. Bundesministerium für Familie S, Frauen und Jugend Ausgeübte Erwerbstätigkeit von Müttern. Erwerbstätigkeit, Erwerbsumfang und Erwerbsvolumen 2010. In.; 2012.

\section{Submit your next manuscript to BioMed Central and we will help you at every step:}

- We accept pre-submission inquiries

- Our selector tool helps you to find the most relevant journal

- We provide round the clock customer support

- Convenient online submission

- Thorough peer review

- Inclusion in PubMed and all major indexing services

- Maximum visibility for your research

Submit your manuscript at www.biomedcentral.com/submit
C Biomed Central 